\title{
Integrals involving Hermite polynomials, generalized hypergeometric series and Fox's $H$-function, and Fourier-Hermite series for products of generalized hypergeometric functions
}

\author{
by Sadhana Mishra (Udaipur)
}

\begin{abstract}
We evaluate an integral involving an Hermite polynomial, a generalized hypergeometric series and Fox's $H$-function, and employ it to evaluate a double integral involving Hermite polynomials, generalized hypergeometric series and the $H$-function. We further utilize the integral to establish a Fourier-Hermite expansion and a double Fourier-Hermite expansion for products of generalized hypergeometric functions.
\end{abstract}

1. Introduction. The object of this paper is to evaluate an integral involving an Hermite polynomial, a generalized hypergeometric series and Fox's $H$-function and utilize it to evaluate a double integral involving Hermite polynomials, generalized hypergeometric series and the $H$-function. We further use the integral to establish a Fourier-Hermite expansion and a double Fourier-Hermite expansion for products of generalized hypergeometric series and the $H$-function.

We also discuss some particular cases of our results and show how they generalize a number of known results of Bajpai $[1,2]$ and Shah [14].

Expansion theorems and Fourier series for generalized hypergeometric functions have wide range of applicability in boundary value problems and applied mathematics. For example, some results of this paper can be used to obtain solutions of the heat conduction problems studied by Bajpai [1], Bhonsle [3], Kampé De Fériet [9] and Shah [15].

The Fourier-Hermite expansions for generalized hypergeometric functions have been given by Bajpai $[1,2]$ and Shah [14]. The references [10, $11,17]$ together with the sources indicated in these references provide a good coverage of the subject. However, nobody so far has attempted to establish double and multiple Fourier-Hermite expansions for generalized hypergeometric functions. 
Fox's $H$-function is a generalization of Meijer's $G$-function [5, pp. 206$222]$; on specializing the parameters, it reduces to almost all special functions appearing in pure and applied mathematics [11, pp. 144-159]. Therefore the results obtained in this paper are of a very general character and hence may encompass several cases of interest. Our results are master formulae from which a large number of results can be derived for Meijer's $G$-function, MacRobert's $E$-function, hypergeometric functions, Bessel functions, Legendre functions, Whittaker functions, orthogonal polynomials, trigonometric functions and others.

It is important to note that operations such as differentiation and integration are easier to perform on the $H$-function than on the original functions, even though the two are equivalent. Thus the $H$-function facilitates the analysis by permitting complex expressions to be represented and handled more simply.

The $H$-function introduced by Fox $[6$, p. 408] will be represented and defined as follows:

$$
\begin{aligned}
H_{p, q}^{u, v}\left[z \mid \begin{array}{c}
\left(a_{p}, e_{p}\right) \\
\left(b_{q}, f_{q}\right)
\end{array}\right] & =H_{p, q}^{u, v}\left[z \mid \begin{array}{c}
\left(a_{1}, e_{1}\right), \ldots,\left(a_{p}, e_{p}\right) \\
\left(b_{1}, f_{1}\right), \ldots,\left(b_{q}, f_{q}\right)
\end{array}\right] \\
& =\frac{1}{2 \pi i} \int_{L} X(s) z^{s} d s
\end{aligned}
$$

where $L$ is a suitable Barnes contour and

$$
X(s)=\frac{\prod_{j=1}^{u} \Gamma\left(b_{j}-f_{j} s\right) \prod_{j=1}^{v} \Gamma\left(1-a_{j}+e_{j} s\right)}{\prod_{j=u+1}^{q} \Gamma\left(1-b_{j}+f_{j} s\right) \prod_{j=v+1}^{p} \Gamma\left(a_{j}-e_{j} s\right)} .
$$

Asymptotic expansion and analytic continuation of the $H$-function was given by Braaksma [4].

The following formulae are required in the proofs:

$$
\int_{-\infty}^{\infty} x^{2 w+m} e^{-x^{2}} H_{m}(x){ }_{P} F_{Q}\left[\begin{array}{l}
A_{P} ; c x^{2 h} \\
B_{Q}
\end{array}\right] d x
$$$$
=2^{m} \sum_{r=0}^{\infty} \frac{\left(A_{P}\right)_{r} c^{r} \Gamma\left(\frac{m+2 w+1}{2}+h r\right) \Gamma\left(\frac{m+2 w+2}{2}+h r\right)}{\left(B_{Q}\right)_{r} r ! \Gamma(w+1+h r)}
$$

$$
=\frac{2^{m} h^{m+w} \prod_{i=0}^{h-1} \Gamma\left(\frac{(m+2 w+1) / 2+i}{2}\right) \prod_{i=0}^{h-1} \Gamma\left(\frac{(m+2 w+2) / 2+i}{2}\right)}{(2 \pi)^{h / 2-1 / 2} \prod_{i=0}^{h-1} \Gamma\left(\frac{w+1+i}{h}\right)}
$$




$$
\times_{P+2 h} F_{Q+h}\left[\begin{array}{l}
\left.A_{P}, \Delta\left(h, \frac{m+2 w+1}{2}\right), \Delta\left(h, \frac{m+2 w+2}{2}\right) ; h^{h} c\right] \\
B_{Q}, \Delta(h, w+1)
\end{array}\right],
$$

where $A_{P}$ denotes $A_{1}, \ldots, A_{P} ; h$ is a positive integer; $\Delta(h, a)$ represents the set of parameters $a / h,(a+1) / h, \ldots,(a+h-1) / h ; P<Q ; w=0,1,2, \ldots$; no one of the $B_{Q}$ is zero or a negative integer.

The integral (1.2) can be easily established by expressing the generalized hypergeometric series in the integrand as in [5, p. 182, (1)] and interchanging the order of integration and summation, which is justified due to the absolute convergence of the integral and summation involved, and evaluating the integral with the help of the integral

$$
\int_{-\infty}^{\infty} x^{2 w+m} e^{-x^{2}} H_{m}(x) d x=\frac{2^{m} \Gamma\left(\frac{m+2 w+1}{2}\right) \Gamma\left(\frac{m+2 w+2}{2}\right)}{\Gamma(w+1)},
$$

which follows from [13, p. 199, (2)] and [5, p. 4, (11)].

An alternative form of the integral can be obtained with further help of $[5$, p. $4,(11)]$ and $[5$, p. $181,(1)]$.

Moreover, we need the formula

$$
\begin{aligned}
& \text { (1.3) } \int_{-\infty}^{\infty} x^{2 w+m} e^{-x^{2}} H_{m}(x){ }_{P} F_{Q}\left[\begin{array}{l}
A_{P} ; c x^{2 h} \\
B_{Q}
\end{array}\right]{ }_{U} F_{V}\left[\begin{array}{l}
E_{U} ; d x^{2 k} \\
G_{V}
\end{array}\right] d x \\
& =2^{m} \sum_{r, t=0}^{\infty} \frac{\left(A_{P}\right)_{r} c^{r}\left(E_{U}\right)_{t} d^{t} \Gamma\left(\frac{m+2 w+1}{2}+h r+k t\right) \Gamma\left(\frac{m+2 w+2}{2}+h r+k t\right)}{\left(B_{Q}\right)_{r} r !\left(E_{V}\right)_{t} t ! \Gamma(w+1+h r+k t)},
\end{aligned}
$$

where in addition to the conditions and notations of (1.2), $k$ is a positive integer; $U<V ; w=0,1,2, \ldots$; no one of the $G_{V}$ is zero or a negative integer.

To derive (1.3), we use the series representation for ${ }_{U} F_{V}$, interchange the order of integration and summation and evaluate the resulting integral with the help of (1.2).

Note 1. By applying the above procedure the integral analogous to (1.3) for the product of $n$ generalized hypergeometric series can be easily evaluated.

We recall the orthogonality property of Hermite polynomials [13, pp. 192-193, (5) \& (6)]:

$$
\int_{-\infty}^{\infty} e^{-x^{2}} H_{m}(x) H_{n}(x) d x= \begin{cases}2^{n} n ! \sqrt{\pi}, & m=n, \\ 0, & m \neq n .\end{cases}
$$


In what follows we also use the following notation: $\lambda$ and $\mu$ are positive numbers, and

$$
\begin{gathered}
\phi(r)=\frac{\left(A_{P}\right)_{r} c^{r}}{\left(B_{Q}\right)_{r} r !}, \quad \psi(t)=\frac{\left(E_{U}\right)_{t} d^{t}}{\left(F_{V}\right)_{t} t !}, \\
F_{1}(x)={ }_{P} F_{Q}\left[\begin{array}{l}
A_{P} ; c x^{2 h} \\
B_{Q}
\end{array}\right], \quad F_{2}(x)={ }_{U} F_{V}\left[\begin{array}{l}
E_{U} ; d x^{2 k} \\
F_{V}
\end{array}\right], \\
H(x)=H_{p, q}^{u, v}\left[z x^{2 \lambda} \mid \begin{array}{l}
\left(a_{p}, e_{p}\right) \\
\left(b_{q}, f_{q}\right)
\end{array}\right],
\end{gathered}
$$

$H_{1}(m, r, t)$

$$
\begin{gathered}
=H_{p+2, q+1}^{u, v+2}\left[z \mid \begin{array}{l}
\left(\frac{1-m-2 w}{2}-h r-k t, \lambda\right),\left(\frac{-m-2 w}{2}-h r-k t, \lambda\right),\left(a_{p}, e_{p}\right) \\
\left(b_{q}, f_{q}\right),(-w-h r-k t, \lambda)
\end{array}\right], \\
H_{2}(x, y)=H_{p, q}^{u, v}\left[z x^{2 \lambda} y^{2 \mu} \mid \begin{array}{c}
\left(a_{p}, e_{p}\right) \\
\left(b_{q}, f_{q}\right)
\end{array}\right],
\end{gathered}
$$

$H_{3}\left(m_{1}, m_{2}, r_{1}, t_{1}, r_{2}, t_{2}\right)$

$$
\begin{gathered}
=H_{p+4, q+2}^{u, v+4}\left[z\left[\begin{array}{l}
\left(\frac{1-m_{1}-2 w_{1}}{2}-h r_{1}-k t_{1}, \lambda\right),\left(\frac{-m_{1}-2 w_{1}}{2}-h r_{1}-k t_{1}, \lambda\right) \\
\left(\frac{1-m_{2}-2 w_{2}}{2}-h r_{2}-k t_{2}, \mu\right),\left(\frac{-m_{2}-2 w_{2}}{2}-h r_{2}-k t_{2}, \mu\right), \\
\left(a_{p}, e_{p}\right) \\
\left(b_{q}, f_{q}\right),\left(-w_{1}-h r_{1}-k t_{1}, \lambda\right),\left(-w_{2}-h r_{2}-k t_{2}, \mu\right)
\end{array}\right],\right. \\
A \equiv \sum_{j=1}^{p} a_{j}-\sum_{j=1}^{q} b_{j}, \quad B \equiv \sum_{j=1}^{v} e_{j}-\sum_{j=v+1}^{p} e_{j}+\sum_{j=1}^{u} f_{j}-\sum_{j=u+1}^{q} f_{j} .
\end{gathered}
$$

2 (i). Integral. We show the following:

$$
\begin{aligned}
\int_{-\infty}^{\infty} x^{2 w+m} e^{-x^{2}} H_{m}(x) F_{1}(x) F_{2}(x) & H(x) d x \\
& =2^{m} \sum_{r, t=0}^{\infty} \phi(r) \psi(t) H_{1}(m, r, t),
\end{aligned}
$$

where $A \leq 0, B>0,|\arg z|<\frac{1}{2} B \pi$, together with the conditions given in (1.2) and (1.3).

P r o of. Expressing the $H$-function in the integrand as a Mellin-Barnes type integral (1.1) and interchanging the order of integrations, which is justified due to the absolute convergence of the integrals involved, yields the 
expression

$$
\frac{1}{2 \pi i} \int_{L} X(s) z^{s} \int_{-\infty}^{\infty} x^{2 w+2 \lambda s+m} H_{m}(x) F_{1}(x) F_{2}(x) d x d s .
$$

Evaluating the inner integral with the help of (1.3), we get

$$
\begin{aligned}
& 2^{m} \sum_{r, t=0}^{\infty} \phi(r) \psi(t) \\
& \times \frac{1}{2 \pi i} \int_{L} X(s) \frac{\Gamma\left(\frac{m+2 w+1}{2}+h r+k t+\lambda s\right) \Gamma\left(\frac{m+2 w+2}{2}+h r+k t+\lambda s\right) z^{s}}{\Gamma(w+1+h r+k t+\lambda s)} d s .
\end{aligned}
$$

Now using (1.1), the value of the integral (2.1) is obtained.

Note 2. The integral analogous to (2.1), involving the product of $n$ generalized hypergeometric series, a Hermite polynomial and the $H$-function can be easily evaluated with the help of the result mentioned in Note 1.

2(ii). Particular cases. Putting $d=0$ in (2.1), we get

$$
\int_{-\infty}^{\infty} x^{2 w+m} e^{-x^{2}} H_{m}(x) F_{1}(x) H(x) d x=2^{m} \sum_{r=0}^{\infty} \phi(r) H_{1}(m, r, 0),
$$

under the conditions of (2.1) with $d=0$.

Note that Singh and Varma [16] evaluated an integral involving the product of an associated Legendre function, a generalized hypergeometric series and the $H$-function [11, p. 40, (2.9.4)] by making use of a finite difference operator $E[13$, p. 33 with $W=1]$. Also, Gupta and Olkha [8] evaluated an integral involving the product of a generalized hypergeometric series and the $H$-function using an integral due to Goyal [7, p. 202].

Srivastava, Gupta and Goyal [17, pp. 61-63] presented some integrals based on the technique of Gupta and Olkha.

In view of the above discussion and $[10,11,17]$ it appears that our integral is more general and new, in addition to the new and simple technique of evaluating such integrals.

Setting $c=0$ in $(2.2)$, we obtain

$$
\int_{-\infty}^{\infty} x^{2 w+m} e^{-x^{2}} H_{m}(x) H(x) d x=2^{m} H_{1}(m, 0,0)
$$

under the conditions of $(2.2)$ with $c=0$.

Putting $2 w+m=2 \omega, m=2 \nu$, in (2.3), using [11, p. 4, (1.2.2)] and simplifying with the help of $(1.1)$ and $[5$, p. $4,(11)]$ leads to a known result due to Bajpai [1, p. 2, (2.1)]. 
For $\lambda$ a positive integer, (2.3) after simplification reduces to a known result given by Shah [14].

On assuming $\lambda$ to be a positive integer, putting $e_{j}=f_{i}=1$ $(j=1, \ldots, p ; i=1, \ldots, q)$, using $[11$, p. 10, (1.7.1)] and simplifying with the help of $(1.1),[5$, p. $4,(11)]$ and $[5$, p. 207, (1)], (2.3) yields another known result (with $t=1$ ) given by Bajpai [2, p. 9, (2.1)].

3(i). Double integral. We show the following:

$$
\begin{aligned}
\int_{-\infty}^{\infty} \int_{-\infty}^{\infty} x^{2 w_{1}+m_{1}} y^{2 w_{2}+m_{2}} & e^{-\left(x^{2}+y^{2}\right)} H_{m_{1}}(x) H_{m_{2}}(y) \\
\times & F_{1}(x) F_{2}(x) F_{1}(y) F_{2}(y) H_{2}(x, y) d x d y \\
= & 2^{m_{1}+m_{2}} \sum_{r_{1}, t_{1}=0}^{\infty} \sum_{r_{2}, t_{2}=0}^{\infty} \phi\left(r_{1}\right) \phi\left(r_{2}\right) \psi\left(t_{1}\right) \psi\left(t_{2}\right) \\
& \times H_{3}\left(m_{1}, m_{2}, r_{1}, t_{1}, r_{2}, t_{2}\right),
\end{aligned}
$$

with conditions of validity the same as for (2.1).

P r o of. Evaluating the $x$-integral with the help of (2.1) and interchanging the order of integration and summation, we get

$$
\begin{aligned}
& 2^{m_{1}} \sum_{r_{1}, t_{1}=0}^{\infty} \phi\left(r_{1}\right) \psi\left(t_{1}\right) \int_{-\infty}^{\infty} y^{2 w_{2}+m_{2}} e^{-y^{2}} H_{m_{2}}(y) F_{1}(y) F_{2}(y) \\
& \times H_{p+2, q+1}^{u, v+2}\left[z y^{2 \mu} \mid \begin{array}{r}
\left(\frac{1-m_{1}-2 w_{1}}{2}-h r_{1}-k t_{1}, \lambda\right),\left(\frac{-m_{2}-2 w_{2}}{2}-h r_{1}-k t_{1}, \lambda\right), \\
\left(a_{p}, e_{p}\right)
\end{array}\right] d y .
\end{aligned}
$$

Now, on applying (2.1) to evaluate the $y$-integral, the value of (3.1) is obtained.

Note 3 . The multiple integral analogous to (3.1) can be easily evaluated by applying the above technique $n-1$ times.

3(ii). Particular cases. Putting $d=0$ in (3.1), we get

$$
\begin{aligned}
\int_{-\infty}^{\infty} \int_{-\infty}^{\infty} x^{2 w_{1}+m_{1}} & y^{2 w_{2}+m_{2}} e^{-\left(x^{2}+y^{2}\right)} H_{m_{1}}(x) H_{m_{2}}(y) \\
& \times F_{1}(x) F_{1}(y) H_{2}(x, y) d x d y \\
= & 2^{m_{1}+m_{2}} \sum_{r_{1}, r_{2}=0}^{\infty} \phi\left(r_{1}\right) \phi\left(r_{2}\right) H_{3}\left(m_{1}, m_{2}, r_{1}, 0, r_{2}, 0\right),
\end{aligned}
$$

under the conditions of (3.1) with $d=0$. 
Setting $c=0$ in $(3.2)$, we obtain

$$
\begin{array}{r}
\int_{-\infty}^{\infty} \int_{-\infty}^{\infty} x^{2 w_{1}+m_{1}} y^{2 w_{2}+m_{2}} e^{-\left(x^{2}+y^{2}\right)} H_{m_{1}}(x) H_{m_{2}}(y) H_{2}(x, y) d x d y \\
=2^{m_{1}+m_{2}} H_{3}\left(m_{1}, m_{2}, 0,0,0,0\right)
\end{array}
$$

under the conditions of (3.2) with $c=0$.

Note 4. The integrals of this section may be employed to establish double and multiple Fourier-Hermite expansions for products of generalized hypergeometric series and the $H$-function.

4(i). Fourier-Hermite series. We show the following expansion:

$$
x^{2 w+m} F_{1}(x) F_{2}(x) H(x)=\frac{1}{\sqrt{\pi}} \sum_{n=0}^{\infty} \frac{1}{n !} H_{n}(x) \sum_{r, t=0}^{\infty} \phi(r) \psi(t) H_{1}(n, r, t),
$$

valid under the conditions of (2.1).

Proof. We have an expansion

$$
f(x)=x^{2 w+m} F_{1}(x) F_{2}(x) H(x)=\sum_{n=0}^{\infty} C_{n} H_{n}(x),
$$

since $f(x)$ is continuous and of bounded variation in $(-\infty, \infty)$.

Multiplying both sides of $(4.2)$ by $e^{-x^{2}} H_{m}(x)$ and integrating with respect to $x$ from $-\infty$ to $\infty$, we have

$$
\int_{-\infty}^{\infty} x^{2 w+m} e^{-x^{2}} F_{1}(x) F_{2}(x) H(x) d x=\sum_{n=0}^{\infty} C_{n} \int_{-\infty}^{\infty} e^{-x^{2}} H_{n}(x) H_{m}(x) d x .
$$

Now using (2.1) and (1.4), we get

$$
C_{m}=\frac{1}{m ! \sqrt{\pi}} \sum_{r, t=0}^{\infty} \phi(r) \psi(t) H_{1}(m, r, t) \text {. }
$$

From (4.2) and (4.3), the Fourier-Hermite expansion (4.1) follows.

4(ii). Particular cases. Putting $d=0$ in (4.1), we obtain

$$
x^{2 w+m} F_{1}(x) H(x)=\frac{1}{\sqrt{\pi}} \sum_{n=0}^{\infty} \frac{1}{n !} H_{n}(x) \sum_{r=0}^{\infty} \phi(r) H_{1}(n, r, 0),
$$

valid under the conditions of (4.1) with $d=0$.

Setting $c=0$ in (4.4), we get

$$
x^{2 w+m} H(x)=\frac{1}{\sqrt{\pi}} \sum_{n=0}^{\infty} \frac{1}{n !} H_{n}(x) H_{1}(n, 0,0),
$$


valid under the conditions of (4.4) with $d=0$.

Putting $2 w+m=2 \omega, m=\nu$, in (4.5), using [11, p. 4, (1.2.2)] and simplifying with the help of (1.1) and [5, p. 4, (11)] yields a known result given by Bajpai [1, p. 3, (4.1)].

For $\lambda$ a positive integer, (4.5) after simplification reduces to a known result due to Shah [14].

On assuming $\lambda$ to be a positive integer, setting $e_{j}=f_{i}=1(j=1, \ldots, p$; $i=1, \ldots, q)$, using $[11$, p. 10, (1.7.1)] and simplifying with the help of (1.1), $[5$, p. $4,(11)]$ and $[5$, p. $207,(1)],(4.5)$ yields another known result given by Bajpai $[2$, p. 11, (3.1)].

5.1. Double Fourier-Hermite series. We establish the following double Fourier-Hermite expansion:

$$
\begin{aligned}
& x^{2 w_{1}+m_{1}} y^{2 w_{2}+m_{2}} F_{1}(x) F_{2}(x) F_{1}(y) F_{2}(y) H_{2}(x, y) \\
& =\frac{1}{\pi} \sum_{n_{1}, n_{2}=0}^{\infty} \frac{1}{n_{1} ! n_{2} !} H_{n_{1}}(x) H_{n_{2}}(y) \\
& \quad \times \sum_{r_{1}, t_{1}=0}^{\infty} \sum_{r_{2}, t_{2}=0}^{\infty} \phi\left(r_{1}\right) \psi\left(t_{1}\right) \phi\left(r_{2}\right) \psi\left(t_{2}\right) H_{3}\left(n_{1}, n_{2}, r_{1}, t_{1}, r_{2}, t_{2}\right),
\end{aligned}
$$

under the conditions of (3.1).

Proof. We have an expansion

$$
\begin{aligned}
f(x, y) & =x^{2 w_{1}+m_{1}} y^{2 w_{2}+m_{2}} F_{1}(x) F_{2}(x) F_{1}(y) F_{2}(y) H_{2}(x, y) \\
& =\sum_{n_{1}, n_{2}=0}^{\infty} A_{n_{1}, n_{2}} H_{n_{1}}(x) H_{n_{2}}(y),
\end{aligned}
$$

since $f(x, y)$ is continuous and of bounded variation in the region $-\infty<$ $x<\infty,-\infty<y<\infty$.

The right hand side of (5.2) is an example of what is called a double Fourier-Hermite series. Instead of discussing the theory, we show a method to find $A_{n_{1}, n_{2}}$ from (5.2). For fixed $x$, we note that $\sum_{n_{1}=0}^{\infty} A_{n_{1}, n_{2}} H_{n_{1}}(x)$ depends only on $n_{2}$; furthermore, it must be the coefficient of the FourierHermite series in $y$ of $f(x, y)$ over $-\infty<y<\infty$.

Multiplying both sides of (5.2) by $e^{-y^{2}} H_{m_{2}}(y)$, integrating with respect to $y$ from $-\infty$ to $\infty$ and using (2.1) and (1.4), we get

$$
x^{2 w_{1}+m_{1}} F_{1}(x) F_{2}(x) \sum_{r_{2}, t_{2}=0}^{\infty} \phi\left(r_{2}\right) \psi\left(t_{2}\right)
$$




$$
\begin{aligned}
& \times H_{p+2, q+2}^{u, v+2}\left[z x^{2 \lambda} \mid \begin{array}{l}
\left(\frac{1-m_{2}-2 w_{2}}{2}-h r_{2}-k t_{2}, \mu\right),\left(\frac{-m_{2}-2 w_{2}}{2}-h r_{2}-k t_{2}, \mu\right), \\
\times\left(a_{p}, e_{p}\right)
\end{array}\right] \\
& =\sqrt{\pi} \sum_{n_{1}=0}^{\infty} A_{n_{1}, m_{2}} m_{2} ! H_{n_{1}}(x) .
\end{aligned}
$$

Multiplying both sides of (5.3) by $e^{-x^{2}} H_{m_{1}}(x)$, integrating with respect to $x$ from $-\infty$ to $\infty$, and using (2.1) and (1.4), we obtain

$$
\begin{aligned}
A_{m_{1}, m_{2}}= & \frac{1}{\pi} \sum_{r_{1}, t_{1}=0}^{\infty} \sum_{r_{2}, t_{2}=0}^{\infty} \phi\left(r_{1}\right) \psi\left(t_{1}\right) \phi\left(r_{2}\right) \psi\left(t_{2}\right) \\
& \times H_{3}\left(m_{1}, m_{2}, r_{1}, t_{1}, r_{2}, t_{2}\right) .
\end{aligned}
$$

Now, (5.1) follows from (5.2) and (5.4).

5(ii). Particular cases. Putting $c=d=0$ in (5.1), we get

$$
\begin{aligned}
& x^{2 w_{1}+m_{1}} y^{2 w_{2}+m_{2}} H_{2}(x y) \\
& \quad=\frac{1}{\pi} \sum_{n_{1}, n_{2}=0}^{\infty} \frac{1}{n_{1} ! n_{2} !} H_{n_{1}}(x) H_{n_{2}}(y) H_{3}\left(n_{1}, n_{2}, 0,0,0,0\right),
\end{aligned}
$$

under the conditions of (5.1) with $c=d=0$.

Note 5. Multiple Fourier-Hermite series analogous to (5.1) can be established on applying the above technique repeatedly.

Note 6 . The Fourier-Hermite series and the double Fourier-Hermite series given in Sections 4 and 5 may further be generalized by using Laplace transform techniques given by Wimp and Luke [18].

No te 7 . The results analogous to our main results (2.1), (3.1), (4.1) and (5.1) involving the $H$-function of several complex variables [17, pp. 251-255] can be derived easily by following the techniques gives in this paper.

I wish to express my sincere thanks to Professor C. M. Joshi, Professor and Head of Department of Mathematics, M. L. Sukhadia University, Udaipur, for his kind guidance during the preparation of this paper.

I am extremely grateful to the referee for his useful comments.

\section{References}

[1] S. D. Bajpai, An integral involving Fox's $H$-function and heat conduction, Math. Ed. (Siwan) 3 (1969), 1-4.

[2] - An expansion formula for Meijer's G-function involving Hermite polynomials, Labdev J. Sci. Tech. Part A8 (1970), 9-11. 
[3] B. R. Bhonsle, Heat conduction and Hermite polynomials, Proc. Nat. Acad. Sci. India Sect. A 36 (1966), 359-360.

[4] B. L. J. Braaksma, Asymptotic expansions and analytic continuations for a class of Barnes integrals, Compositio Math. 15 (1963), 239-341.

[5] A. Erdélyi, Higher Transcendental Functions, Vol. 1, McGraw-Hill, New York 1953.

[6] C. Fox, The $G$ and $H$-functions as symmetrical Fourier kernels, Trans. Amer. Math. Soc. 98 (1961), 395-429.

[7] G. K. Goyal, An integral involving H-function, Proc. Nat. Acad. Sci. India Sect. A 39 (1969), 201-203.

[8] K. C. Gupta and G. S. Olkha, Integrals involving the products of generalized hypergeometric functions and Fox's H-function, Univ. Nac. Tucumán Rev. Ser. A 19 (1969), 205-212.

[9] J. Kampé De Fériet, Heat conduction and Hermite polynomials, Bull. Calcutta Math. Soc., Golden Jubilee Commemoration Volume (1958-59), 103-104.

[10] A. M. Mathai and R. K. Saxena, Generalized Hypergeometric Functions with Applications in Statistics and Physical Sciences, Lecture Notes in Math. 348, Springer, Berlin 1973.

[11] - - - The H-Function with Applications in Statistics and Other Disciplines, Wiley Eastern Ltd., New Delhi 1978.

[12] L. M. Milne-Thomson, The Calculus of Finite Differences, Macmillan, London 1933.

[13] E. D. Rainville, Special Functions, McGraw-Hill, New York 1960.

[14] M. Shah, On some results on the $H$-function involving Hermite polynomials, J. Natur. Sci. Math. 9 (1969), 223-233.

[15] - , Heat conduction, generalized Meijer's function and Hermite polynomials, Comment. Math. Univ. St. Paul. 19 (1970), 81-94.

[16] F. Sing h and R. C. V arma, Application of E-operator to evaluate a definite integral and its application in heat conduction, J. Indian Math. Soc. (N.S.), 36 (1972), 325332 .

[17] H. M. Srivastava, K. C. Gupta and S. P. Goyal, The H-function of One and Two Variables with Applications, South Asian Publ., New Delhi 1982.

[18] J. Wimp and Y. L. Luke, Expansion formulae for generalized hypergeometric functions, Rend. Circ. Mat. Palermo 11 (1962), 351-366.

V.B.R.I. POLYTECHNIC

VIDYA-BHAWAN RURAL INSTITUTE

UDAIPUR, INDIA 